\title{
Modifizierte Schaumsklerosierung: offene Fragen
}

Tiwari A et al. Re: "Catheter Foam Sclerotherapy of Great Saphenous Vein, with Peri-saphenous Tumescence and Saphenous Irrigation" - Is modified Catheter Foam Sclerotherapy a Step Back in the Evolution of Endovenous Ablation for Varicose Veins? Eur J Vasc Endovasc Surg. 2018;55:290-291. doi:10.1016/j. ejvs.2017.10.016

Die Gefäßexperten beziehen sich auf einen Studienbericht von Cavezzi et al. in derselben Ausgabe (Eur J Vasc Endovasc Surg. 2017;54:629-635. doi:10.1016/j. ejvs.2017.08.004). Die vorgestellte Methode bestand in einer Schaumsklerosierung der varikösen V. saphena magna (GSV), die Katheter-gestützt, mit Tumeszenz und Flush erfolgte. Die Okklusionsrate betrug nach 3 Jahren 89,4\% und übertraf damit frühere Resultate der sonographisch geleiteten GSV-Schaumsklerosierung (UGFS).

Die Kommentatoren befürchten, dass die Technik einen Rückschritt in der Behandlung von GSV-Varizen darstellt. Es gehe nicht nur um hohe Verschlussraten im Vergleich zum Goldstandard endothermale Ablation. Weniger schmerzhafte Eingriffe, eine höhere Patientenzufriedenheit und weniger Komplikationen seien die wesentlichen Endpunkte. Diese Ideale waren die Basis für die Entwicklung von Methoden ohne Hitzeeinwirkung und ohne Tumeszenz. Die modifizierte Schaumsklerosierung treffe ins Herz dieser Ziele: Die Notwendigkeit der Tumeszenz, häufigere Nadelstiche, ein höheres Hämatomrisiko, mehr Schmerzstimuli und eine längere Interventionsdauer belasten den Patienten. Darüber hinaus ergaben sich technische und methodische Fragen. Der Katheter wurde $5 \mathrm{~cm}$ von der saphenofemoralen Junktion platziert, woraus sich das Risiko einer höheren Rekanalisationsrate ergebe. Bei 3 Patienten gelang die Platzierung wegen Gefäßdilatationen nicht. Offen blieb, warum nicht ein Versuch mit einem anderen Kathetertyp erfolgte. Unklar schien auch die Phlebektomie von Seitenästen anstelle einer niedrig dosierten UGFS. 3,4\% der Patienten berichteten über Parästhesien. Die von Leitlinien empfohlene postinterventionelle Kontrolle nach 1 Woche fehlte ebenso wie eine Vergleichsgruppe.

Tiwari et al. glauben, dass die Verkleinerung des Gefäßdurchmessers, der geringere Blutgehalt und die präzisere Schaumapplikation die Studienergebnisse nicht allein erklären. Neben der Stabilität des Sklerosierungsmittels sei der Tumeszenzprozess bedeutsam. Durch die mögliche Verletzung der Tunica media mit einem „escape“ der Verödungssubstanz könne die Apoptose von Adventitiazellen einen direkten Venenverschluss begünstigen.

Dr. med. Susanne Krome, Melle 